\title{
Introduction to the Competitive Strategy, Economics and IS Mini-Track
}

\author{
Eric K. Clemons \\ University of Pennsylvania \\ clemons@wharton.upenn.edu
}

\author{
Robert J. Kauffman \\ Singapore Management Univ. \\ rkauffman@smu.edu.sg
}

\author{
Thomas A. Weber \\ École Polytechnique \\ Fédérale de Lausanne \\ thomas.weber@epfl.ch
}

For the past 25 years, this mini-track has offered a forum for new ideas on IT and competitive strategy informed by economic theory and research methods. This year, it includes peer-reviewed and "for presentation only" papers over two days, beginning on the Tutorial and Workshop day. The peer-reviewed papers cluster in two sessions.

The first session's theme is "Trust and Security." The first paper is "The Role of Trust in Successful ECommerce Websites in China: Field Observations and Experimental Studies," whose authors are E.K. Clemons, F. Jin, J. Wilson, F. Ren, C. Matt, T. Hess and N.S. Koh. They test hypotheses on the precursors of e-commerce trust in different cultural and national settings. Their hypotheses cover seller reputation and promises, third-party guarantees, consumer perceptions of risk in Internet-based selling, and the efficacy of mechanisms for consumer risk reduction. Their work was carried out in four different countries.

The second paper is "Quality Competition in the Software Security Market," by D. Dey, A. Lahiri and G. Zhang. They explore reasons for the lack of product differentiation in the security software market, where there are many competitors, high prices and low coverage. They use a quantitative model to theorize about different outcomes that may occur under monopoly, duopoly and oligopoly market structures. They find that there is a negative network effect related to security software, which arises as market coverage increases and the likelihood of indirect attacks from unprotected computers decreases. This also tends to diminish producer incentives to differentiate their products and services. The research also offers insights on the uniqueness of the market structure for the sale of security software.

The session closes with J. Kwon and M.E. Johnson's paper, entitled "Healthcare Security Strategies for Regulatory Compliance and Data Security." They distinguish among IT security resources, functional auditing and managerial capabilities to assess information security and compliance in 250 healthcare organizations. They use econometrics models for the probabilities of observing data breach events and seven different levels of regulatory compliance. They find that mitigating the severity of data breaches depends more on the extent of the security resources and the prevention capabilities of healthcare organizations, than healthcare organization regulatory compliance. In fact, greater compliance leads to the discovery of more information security breach events.

The mini-track's second session covers issues that deal with "Economics and Electronic Markets." The first paper is entitled "Does It Pay Off to Bid Aggressively? An Empirical Study," by P. Herrmann, D. Kundisch and M. Rahman. They evaluate the efficacy of signals related to aggressive bidding in online payper-bid auctions, a type of ascending price auctions. Based on about 7,000 auctions, and 600,000 bids from 2,600 bidders, their exploratory model estimates the number of auction participants and total bids for the possible impact of aggressive bids, as well as other auction-level covariates. Aggressive bidding, they find: (1) encourages more participants and more bids overall; but (2) diminishes the probability that individual bidders will win. The implication is aggressive bidding is not the way to deter competitors from participating in an electronic auction; if anything, it stimulates them to compete harder to win.

The last paper investigates the dynamics of information transmission related to the wisdom of crowds. L. Qiu, H. Rui and A.B. Whinston consider a question on "Information Exchange in Prediction Markets: Do Social Networks Promote Forecast Efficiency?" They use game theory analysis - more specifically, an incomplete information game with a symmetric Bayes-Nash market equilibrium - to show that social network-provided information may not enhance prediction market forecast accuracy, especially when information acquisition costs are high. This result suggests the participants' use of a threshold strategy. Information acquisition decreases as a participant's friends and network density increase also. The authors apply their ideas to prediction markets for social networks and securities trading. 